\title{
Estimation of Viscous Damping Parameters of Fibre Reinforced Plastic Plates using Finite Element Model Updating
}

\author{
Subhajit Mondal and Sushanta Chakraborty \\ Department of Civil Engineering, Indian Institute of Technology Kharagpur, Kharagpur-721 302, India.
}

(Received 8 April 2015; accepted 16 February 2016)

\begin{abstract}
A combined numerical-experimental technique has been implemented using the finite element model updating technique to estimate a set of proportional viscous damping parameters for determining the global responses of fibre reinforced plastic (FRP) plates over a chosen frequency range of interest. The experimentally determined frequencies and mode shapes are used to update the homogenised anisotropic in-plane material constants, before estimating the damping parameters from the directly observed frequency response functions (FRFs). Gradient based inverse sensitivity method has been implemented for the parameter estimation. Existing FRP structures may degrade considerably due to environmental effects over the long period of existence-changing the material and damping properties significantly compared to their initial values after fabrication - thus requiring updating. The estimated viscous damping parameters using the current technique reproduces higher values of modal damping factors for FRP plates. For nonviscous damping, estimation of a set of relaxation factors produces a more realistic estimate of modal damping factors. Increased value of the relaxation factors make the model coincide with the viscous one. A numerically simulated plate problem has been presented along with the experimental validation.
\end{abstract}

\section{INTRODUCTION}

Dynamical responses of Fibre Reinforced Plastic (FRP) plates are functions of geometry, material properties, existing boundary conditions, and damping. The initial material properties used for modelling the dynamical responses of such plates come from the material characterisation tests on separately prepared samples. However, development of an accurate damping matrix is far more difficult, even at the initial stage of existence of the plate, because the global damping matrix is usually formulated directly from the global stiffness and mass information. It is extremely difficult to form a damping matrix from constituent level damping parameters, taking care of all the complex individual phenomena and their combinations, such as material damping, joint friction, and environmental effects. Gaul provided excellent guidelines to understand the damping of materials and structural members, including laminated parts. ${ }^{1}$ Making assumptions about small damping allows linear damping models to approximate the actual nonlinear damping behaviour. In proportional damping-where the viscous effects are dominant-the damping matrix is formed by weighted sum of mass and stiffness matrices. ${ }^{2}$ Thus, proportional damping or Rayleigh damping is mathematically expressed as

$$
C=a_{0} M+a_{1} K .
$$

Here, $M, C$, and $K$ are the mass, viscous proportional damping, and stiffness matrices of the FRP plate considering the plate as a multiple degrees of freedom system. The coefficients $a_{0}$ and $a_{1}$ are the Rayleigh damping coefficients. The Rayleigh damping model has enjoyed an almost universal acceptance because the modal transformation of the proportional damping matrix - with respect to the undamped normal mode shapesproduces a diagonal damping matrix. However, a more generalised viscous proportional damping matrix has been proposed by Caughy and Caughey and Kelly 3,4

$$
C=M \sum_{n=0}^{N-1} a_{n}\left[M^{-1} K\right]^{n} .
$$

In fact, the value of $n$ can be chosen as an integer between $-\infty$ and $+\infty$, but practically this is taken very low (e.g., $n=2$ ) for Rayleigh damping. 5 Woodhouse emphasised the importance of accurate predictions of physical mechanisms for estimating an appropriate damping matrix. ${ }^{6}$ Adhikari incorporated frequency variations of damping factors to determine a generalised proportional damping model. ${ }^{7}$ Adhikari and Phani obtained a generalised proportional damping matrix from a single driving point frequency response function (FRF) only. ${ }^{8}$

Several investigators have used measured FRFs for damping identification. ${ }^{9-13}$ Arora et al. implemented a two-step damping identification methodology in which the stiffness and mass matrices are updated first from the measured FRFs, followed by identification of the damping matrix. ${ }^{14}$ Friswell identified a generalised viscous proportional damping matrix along with the stiffness matrix from measured FRFs. ${ }^{15}$ More recently, Pan and Wang used a FRF based iterative model updating approach to identify an exponential damping model. ${ }^{16}$ Kausel proposed methodology to construct families of nonproportional damping matrices. ${ }^{17}$

Extensive investigations have been carried out by Dalenbring on laminated aluminium and Plexiglas plates, constrained viscoelastic layers of aluminium and carbon fibre epoxy laminate, and bonded aluminium polymethyl methacrylate plates to identify damping parameters. ${ }^{18-21}$ Dovstam formulated a linear three-dimensional material damping modelling technique using an augmented Hooke's law (AHL) in the frequency domain, and recently investigated interface damping in built-up structures. ${ }^{22,23}$ 
Literature related to modelling of damped FRP structures is limited. Crocker and $\mathrm{Li}$ presented reviews on damping of composites including sandwich composites. ${ }^{24}$ Gelman et al. used a FRF-based diagonalization of damping matrix, Akrout et al. investigated the vibroacoustic behaviour of thin filmlaminated glass panels in the presence of fluid layers, and $\mathrm{Wu}$ and Huang studied the natural frequency and the damping of laminated composites plates. ${ }^{25-27}$

Identification of damping using a finite element model updating is an active area of research. ${ }^{28}$ All existing methods of damping estimation usually assume availability of accurate mass and stiffness matrices; in reality, these requirements are hard to achieve for existing FRP structures, since uncertainties will grow both for stiffness and damping parameters as the structure deteriorates over time. As a result, most of the proposed techniques in literature for estimation of damping are not directly applicable to real existing structures unless a stiffness identification exercise is carried out prior to or in conjunction with the damping identification. Since the phenomenon of damping is complex, a proper, regularised, well-posed inverse approach is the only solution to forming a reliable damping matrix from measured modal or FRF responses.

The objective of the present investigation is to apply a model updating technique to estimate a set of globally equivalent viscous damping parameters and stiffness parameters for pultruded FRP plates using the Inverse Eigensensitivity Method (IEM) so that the predicted responses matches exactly with observed responses. ${ }^{29}$ The procedure includes detailed experimental modal testing and subsequent data analysis, precise finite element modelling with appropriate discretization, correlating finite element results to the experiment, and updating selected parameters using a robust gradient-based optimization technique. ${ }^{30}$ The methodology is established by a numericallysimulated example, followed by a real experimental case study on a pultruded FRP plates under free-boundary conditions. A set of relaxation parameters has been defined to model nonviscous damping for FRP plates. Increased values of these relaxation factors make the model coincide with the viscous one.

\section{MATHEMATICAL FORMULATION}

\subsection{Estimation of Viscous Damping Parameters}

The equation of motion of the FRP plate in time domain can be written as

$$
M \ddot{x}(t)+C \dot{x}(t)+K x(t)=f(t) .
$$

Here, $x(t)$ is the displacement vector. The undamped eigenvalues and eigenvectors are computed from the eigensolutions of the equation

$$
K \phi=\omega^{2} M \phi
$$

The undamped eigenvalues, eigenvectors and the modal damping factors can be related through the expression of accelerance FRFs as

$$
H_{p q}(\omega)=-\omega^{2} \sum_{n=1}^{N} \frac{\phi_{p n} \phi_{q n}}{\left(\omega_{n}-\omega+2 i \omega \omega_{n} \xi_{n}\right)} ;
$$

where $H_{p q}$ is acceleration response at point $p$ due to excitation at point $q .{ }^{31}$ The modal damping factor $\xi_{n}$ for $n^{\text {th }}$ mode of the FRP plate can be expressed as

$$
\xi_{n}=\frac{1}{2}\left(\frac{a_{0}}{\omega_{n}}+a_{1} \omega_{n}+a_{2} \omega_{n}^{3}+\ldots+a_{N-1} \omega_{n}^{2 N-3}\right) .
$$

For proportional damping, $N$ can be taken as 2 . Thus, Eq. (6) reduces to

$$
\xi_{n}=\frac{1}{2}\left(\frac{a_{0}}{\omega_{n}}+a_{1} \omega_{n}\right) .
$$

Here, $a_{0}$ and $a_{1}$ are the Rayleigh damping coefficients. When $N>2$ in Eq. (6), the damping matrix is a fully populated matrix, ${ }^{32}$ whereas the stiffness and mass matrices still remain sparse and banded. Substituting the values of Rayleigh damping coefficients, Eq. (5) can be expressed as

$$
H_{p q}(\omega)=-\omega^{2} \sum_{n=1}^{N} \frac{\phi_{p n} \phi_{q n}}{\left(\omega_{n}-\omega+i \omega\left(a_{1} \omega^{2}+a_{0}\right)\right)} .
$$

Out of all three system matrices, the mass matrix has less uncertainty. Moreover, the magnitude of the coefficients of the damping matrix is an order lower in comparison to the mass and stiffness matrices in a consistent formulation. Thus, for estimating the damping parameters of the FRP plate, a twostage model updating approach needs to be implemented by updating the stiffness matrix in the first phase, then updating modal damping coefficients - only parameters $a_{1}$ and $a_{0}$-in the damping matrix during the second phase.

The objective function can be formed as the summation of the weighted differences of the measured and computed FRFs (used to estimate damping parameters) or the frequencies and mode shapes (used to estimate stiffness parameters) of the FRP plate. The objective function can be expressed as

$$
\begin{aligned}
& E=\sum_{p=1}^{n} w_{p p}\left\|H_{\text {exp }}-H_{\mathrm{nu}}\right\|^{2} ; \quad \text { or } \\
& E=\sum_{p=1}^{n} w_{p p}\left\|(\omega, \phi)_{\exp }-(\omega, \phi)_{\mathrm{nu}}\right\|^{2} ;
\end{aligned}
$$

where $w_{p p}$ are the weights and $p$ is the number of frequencies or FRFs considered sensitive enough with respect to the elastic parameters or modal damping coefficients. The sensitivities of the frequencies, mode shapes, or the FRFs with respect to the material parameters or damping parameters are computed using first-order finite difference approximation

$$
S=\left[\frac{\partial(\omega, \phi \text { or } H)}{\partial r_{n}}\right] \text {. }
$$

Here, $r_{n}$ is the material or damping parameters for which sensitivity is calculated. The relationship between changes in measured modal properties (e.g., frequencies, mode shapes, or FRFs) and the changes in the parameters to be updated (e.g., material constants, $a_{0}$, and $a_{1}$ ) of the FRP plate can be related through the first-order sensitivity matrix

$$
\{\Delta f\}=[S]\{\Delta r\} .
$$

Suitable changes $(\Delta r)$ are made to the initial guesses of the parameters within reasonably selected bounds, and the finite element model of the pultruded FRP plate is updated iteratively, 
minimizing the objective function in a weighted least-square sense. Such changes implement the IEM

$$
\{r\}_{p+1}=\{r\}_{p}+\{\Delta r\}_{p}
$$

The similarity of experimental modes with the numerically computed modes is quantitatively verified during each step of iteration through Modal Assurance Criteria (MAC); Values close to 1 indicate good correlation. ${ }^{33}$ The experimental FRFs are compared with the numerical FRFs using Signature Assurance Criteria (SAC). ${ }^{31}$ The above correlations are vector correlations, whereas the FRF magnitudes are compared using Cross Signature Assurance Criteria (CSAC) and Cross Signature Scale Factor (CSF). ${ }^{33,34}$

\subsection{Estimation of Relaxation Factors from Updated Viscous Damping Parameters}

For nonviscous damping, the equation of motion (Eq. (3)) can be written as ${ }^{35}$

$$
M \ddot{x}(t)+\int_{0}^{t} G(t-\tau) \dot{x}(\tau) d \tau+K x(t)=0 .
$$

Here, the dissipative force depends on the time-history of motion. This can be represented by a convolution integral between the velocities and a decaying kernel function. One promising expression for $G(t)$ is exponential damping model ${ }^{35}$

$$
G(t)=C \mu e^{-\mu t} .
$$

Here, $C$ is the damping matrix with real coefficients and $\mu$ is the relaxation factor. The viscously-damped system can be expressed as a special case of exponential nonviscous damping when $\mu \rightarrow \infty$. The Laplace transformation of the dynamical equation can be expressed as

$$
s^{2} M z+s L(s) z+K z=0 .
$$

Here, $L(s)$ is the Laplace transformation of $G(t), z$ is the complex mode, and $s$ is the complex frequency of the FRP plate. Assuming small damping and first-order perturbation theory, the $q^{\text {th }}$ complex natural frequency and mode shape can be approximated as 6

$$
\begin{aligned}
\lambda_{q} & = \pm \omega_{q}+i \frac{L_{q q}^{\prime}(\omega)}{2} \\
z_{q} & =\phi_{q}+i \sum_{\substack{n=1 \\
n \neq q}}^{N} \frac{\omega_{q} L_{n q}^{\prime}(\omega) \omega_{q}}{\left(\omega_{q}^{2}-\omega_{n}^{2}\right)} \phi_{n}
\end{aligned}
$$

The imaginary part of the above equation can be written as ${ }^{16}$

$$
\begin{aligned}
L_{n l}^{\prime}\left(\omega_{q}\right) & =\phi_{n}^{T} L\left(\omega_{q}\right) \phi_{l} ; \\
L_{n l}^{\prime}\left(\omega_{q}\right) & =\frac{\mu C_{n l}^{\prime}}{\left(\mu+i \omega_{q}\right)}=\frac{\mu\left(\mu-i \omega_{q}\right) C_{n l}^{\prime}}{\left(\mu^{2}+\omega_{q}^{2}\right)} \\
& =\frac{\mu^{2} C_{n l}^{\prime}}{\left(\mu^{2}+\omega_{q}^{2}\right)}-i \frac{\mu \omega_{q} C_{n l}^{\prime}}{\left(\mu^{2}+\omega_{q}^{2}\right)}
\end{aligned}
$$

where

$$
C_{n l}^{\prime}=\phi_{n}^{T} C \phi_{l}
$$

In the above equation, phi is the real part of the complex mode shapes. Assuming that

$$
G(t)=\left(a_{0} M+a_{1} K\right) \mu e^{-\mu t}=C_{0} \mu e^{-\mu t}+C_{1} \mu e^{-\mu t} ;
$$

the Laplace transform of the equation can be written as ${ }^{16}$

$$
\begin{aligned}
L_{n l}^{\prime}\left(\omega_{q}\right)= & \left\{\frac{\mu_{0}^{2} C_{0, n l}^{\prime}}{\left(\mu_{0}^{2}+\omega_{q}^{2}\right)}+\frac{\mu_{1}^{2} C_{1, n l}^{\prime}}{\left(\mu_{1}^{2}+\omega_{q}^{2}\right)}\right\}- \\
& i\left\{\frac{\mu_{0} \omega_{q} C_{0, n l}^{\prime}}{\left(\mu_{0}^{2}+\omega_{q}^{2}\right)}+\frac{\mu_{1} \omega_{q} C_{1, n l}^{\prime}}{\left(\mu_{1}^{2}+\omega_{q}^{2}\right)}\right\}
\end{aligned}
$$

Here

$$
\begin{aligned}
& C_{0, n l}^{\prime}=a_{0} \phi_{n}^{T} M \phi_{l} \\
& C_{1, n l}^{\prime}=a_{1} \phi_{n}^{T} M \phi_{l}
\end{aligned}
$$

$$
\begin{aligned}
\lambda_{q}= \pm \omega_{q}+ & \left\{\frac{a_{0}}{2} \frac{\mu_{0} \omega_{q}}{\left(\mu_{0}^{2}+\omega_{q}^{2}\right)}+\frac{a_{1} \omega_{q}^{2}}{2} \frac{\mu_{1} \omega_{q}}{\left(\mu_{1}^{2}+\omega_{q}^{2}\right)}\right\}+ \\
& i\left\{\frac{a_{0}}{2} \frac{\mu_{0}^{2}}{\left(\mu_{0}^{2}+\omega_{q}^{2}\right)}+\frac{a_{1} \omega_{q}^{2}}{2} \frac{\mu_{1}^{2}}{\left(\mu_{1}^{2}+\omega_{q}^{2}\right)}\right\} .
\end{aligned}
$$

The complex natural frequency corresponding to the elastic modes can be written as

$$
\lambda_{q}=\omega_{q}+i \omega_{q} \xi_{q}
$$

Here, $\xi_{q}$ is the modal damping of $q^{\text {th }}$ mode. Comparing the imaginary parts of the above two equations, the value of the modal damping factor can be correlated to the relaxation parameter as shown ${ }^{16}$

$$
\xi_{q}=\frac{a_{0}}{2 \omega_{q}} \frac{1}{\left(1+\frac{\omega_{q}^{2}}{\mu_{0}^{2}}\right)}+\frac{a_{1} \omega_{q}}{2} \frac{1}{\left(1+\frac{\omega_{q}^{2}}{\mu_{1}^{2}}\right)} ;
$$

where $a_{0}$ and $a_{1}$ are values of updated damping parameters with respect to the measured FRFs. When the value of $\mu_{0}$, $\mu_{1}$ are comparatively higher, then the exponential damping closely matches with the Rayleigh damping.

\section{NUMERICALLY SIMULATED EXAMPLE}

Consider a rectangular FRP composite plate of dimensions $400 \mathrm{~mm} \times 300 \mathrm{~mm}$ and with a thickness of $10 \mathrm{~mm}$. This numerically-simulated example deals with the estimation of the in-plane stiffness properties and global damping parameters of a pultruded FRP composite plate. A $12 \times 12$ mesh division using the isoparametric element (S8R) in ABAQUS was found to be adequate for the convergence of eigenproperties within selected frequency ranges, and is used throughout this present investigation. The experimental frequency, mode shapes, and FRF data are generated using the same finite element model. The in-plane material elastic constants are taken as $E_{x}=35 \mathrm{GPa}, E_{y}=30 \mathrm{GPa}, G_{x y}=5 \mathrm{GPa}$; the Poission's ratio is taken as $v_{x y}=0.17$. Here, $x$ and $y$, respectively, are the longitudinal and transverse in-plane directions of the FRP plate. The data is similar to the actual experimental case study reported later. The transverse shear moduli, $G_{x z}$ and $G_{y z}$ both are assumed to be $5 \mathrm{GPa}$. The direction $z$ implies direction 
Table 1. Numerically simulated "experimental" frequencies and assumed modal damping factors.

\begin{tabular}{|c|c|c|}
\hline Mode & Frequency $(\mathrm{Hz})$ & Modal damping factor (\%) \\
\hline 1 & 159.56 & $2.0 \mathrm{E}-2$ \\
2 & 286.18 & $0.75 \mathrm{E}-2$ \\
3 & 431.35 & $1.5 \mathrm{E}-2$ \\
4 & 553.60 & $1.0 \mathrm{E}-2$ \\
5 & 631.44 & $1.1 \mathrm{E}-2$ \\
6 & 793.82 & $1.20 \mathrm{E}-2$ \\
7 & 898.91 & $0.8 \mathrm{E}-2$ \\
8 & 917.55 & $0.8 \mathrm{E}-2$ \\
\hline
\end{tabular}

Table 2. Initial value of Rayleigh damping parameters for the numerical model.

\begin{tabular}{||c|c|c|c|c||}
\hline & $\begin{array}{c}\text { Mode considered } \\
\text { for average values } \\
\text { of } \omega_{1} \text { and } \zeta_{1}\end{array}$ & $\begin{array}{c}\text { Mode considered } \\
\text { for average values } \\
\text { of } \omega_{1} \text { and } \zeta_{1}\end{array}$ & $a_{0}$ & $a_{1}$ \\
\hline Trial_1 & 1,2 & 3,4 & 3.91 & $3.71 \mathrm{E}-5$ \\
Trial_2 & $1,2,3$ & $4,5,6$ & 6.28 & $1.92 \mathrm{E}-5$ \\
Trial_3 & $1,2,3,4$ & $5,6,7,8$ & 6.76 & $1.40 \mathrm{E}-5$ \\
Trial_4 & - & - & 40 & $8.0 \mathrm{E}-6$ \\
Trial_5 & - & - & 50 & $15.0 \mathrm{E}-6$ \\
\hline
\end{tabular}

perpendicular to the plane of the plate. Since the in-plane Poission's ratio can only be estimated properly when sufficient numbers of torsional modes are included as information, this factor was removed from scope of the experimental investigation. The mass density is assumed to be $1500 \mathrm{kgm}^{-3}$. The assumed experimentally measured modal damping parameters and natural frequencies are given in Table 1.

The parameter estimation exercise was carried out using the inverse eigensensitivity method (IEM) to estimate the material parameters first. The convergences from a few initial values to the correct material constants are shown in Fig. 1. As expected, changes in modal damping values had little effect on the estimation of the in-plane material constants.

In Fig. 1, s_40,37,10 is a set of initial values of the material constants $E_{x}=40 \mathrm{GPa}, E_{y}=37 \mathrm{GPa}$, and $G_{x y}=10 \mathrm{GPa}$ used in the model updating exercise. The solutions were unique in all cases.

The estimate of the damping coefficients $a_{0}$ and $a_{1}$ is sensitive to the FRF magnitudes. Simulated "experimental" frequencies and modal damping parameters from Table 1 were used to choose sets of initial values of $a_{0}$ and $a_{1}$ for use in the model updating program. Equation (7) gives a set of initial values of $a_{0}$ and $a_{1}$, which differ considerably depending upon the modes selected, as shown in Table 2. ${ }^{32}$ The first three sets show the variations of these two parameters due to the incorporation of varying number of modes - at most, eight modes here. To check the robustness of this FRF based inverse algorithm, two additional arbitrary sets of $a_{0}$ and $a_{1}$ values were chosen, which did not immediately correspond to any combination of modes; this is shown in Table 2.

Figure 2, shows the convergence curves for both the parameters which are found to be monotonic and unique. The values are $a_{0}=2.53 \mathrm{E} 1$ and $a_{1}=4.27 \mathrm{E}-6$ respectively. The updated mass-proportional damping coefficient converged to a higher value while the stiffness proportional damping coefficient converged to a somewhat lower value. Similar instances have been reported in current literature, demonstrating that a higher value of mass-proportional damping coefficients indicates a trend towards more viscous damping. ${ }^{16}$ Also, similar observations have been made for FRP beams. ${ }^{30}$

The regenerated FRF curves-as shown in Fig. 3- (a) Convergence of $E_{x}$

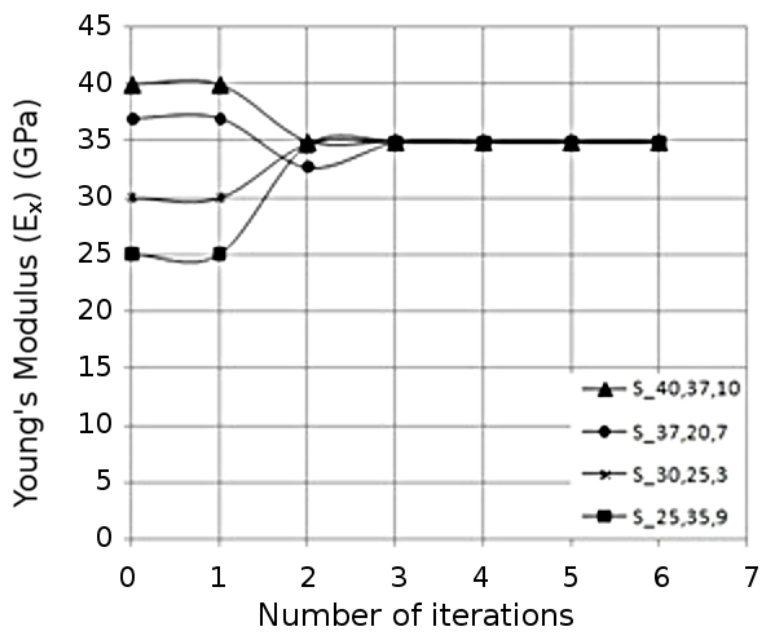

(b) Convergence of $G_{x y}$

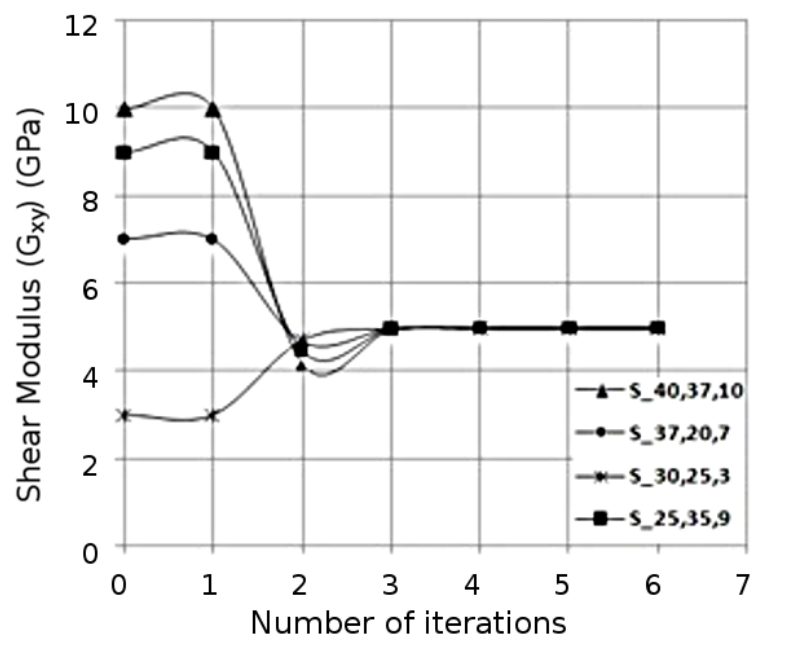

Figure 1. Typical convergence curves for material parameters from selected initial values.

continually matched as additional modes were added into the simulation, indicating that the updated parameters of the identified damping matrix are globally representative. Thus, the response prediction of the plate became much more accurate. The degree of improvement was judged by comparing improved values of MAC and SAC between the simulated "experimental" observation and the observations made using the updated model.

Figure 4 shows the FRF curve with $2 \%$ random noise in FRF data; here, the damping parameters were updated to the values $a_{0}=2.55 \mathrm{E} 1$ and $a_{1}=4.3 \mathrm{E}-6$, which can be compared with the previous values obtained in the FRF data without noise. Thus, the noise sensitivity of the algorithm was observed to be low.

\section{EXPERIMENTAL INVESTIGATION}

A rectangular FRP composite plate of the same size$400 \mathrm{~mm} \times 300 \mathrm{~mm}$, with a thickness of $10 \mathrm{~mm}$, as described in the numerically simulated example-was fabricated out of woven roving glass fibres and epoxy matrix using the pultrusion process. In fact, the plate has been extracted from a 


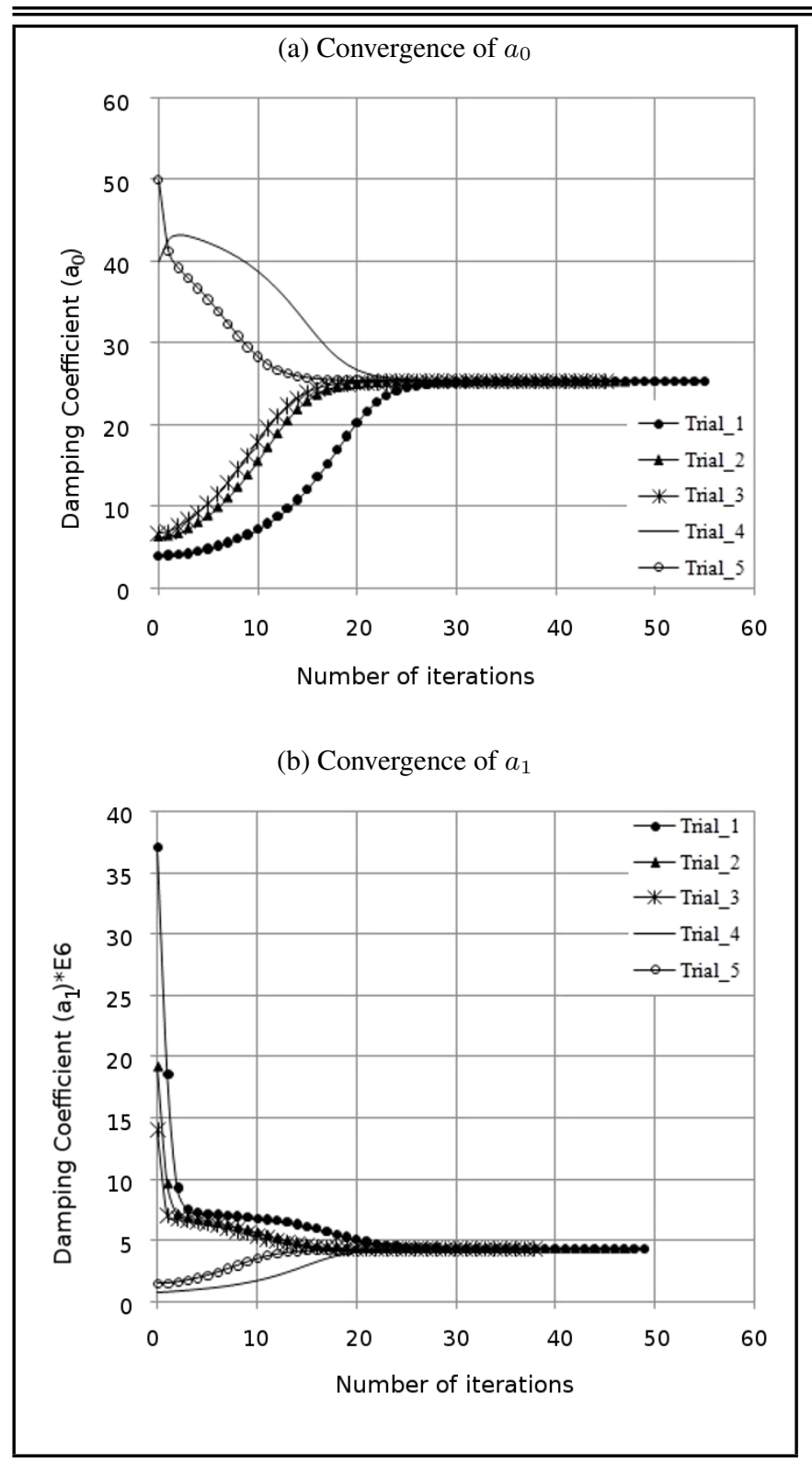

Figure 2. Convergence curves for the damping coefficients.

much bigger plate fabricated for the purpose of experimentation on beam samples as well as for the plate. ${ }^{30}$ The average thickness and the mass density of the plate was $10.12 \mathrm{~mm}$ and $2012 \mathrm{kgm}^{-3}$ respectively. ${ }^{30}$ Modal testing was carried out on the FRP plate under free-boundary conditions, using impact excitation imparted through an impact hammer; Responses were picked up by an accelerometer. The Frequency Response Functions (FRFs) were directly measured, and frequencies and mode shapes were extracted using modal analysis software MEScope. Such modal testing is standard in current practice-details of similar modal testing on pultruded FRP composite beam may be obtained from investigations carried out earlier. ${ }^{30,31}$ Figure 5(a) shows the modal test setup, in which the free boundary condition was realised by suspending the FRP plate using soft elastic strings from a rigid support. Figure 5(b) shows the position of the accelerometer (point 16), and a few selected points of excitations of the plate with the impact hammer. All 49 points were used as excitation points.

The finite element modelling of the plate was performed using the same shell element (S8R) with adequate discretization (a) Comparison of FRF obtained using modal damping and FRF obtained using damping parameters (Trial_1)

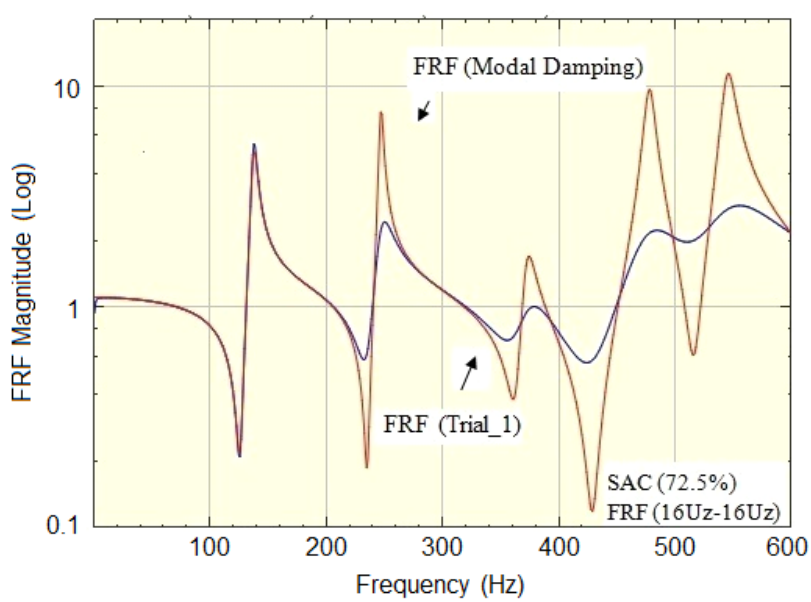

(b) Comparison of FRF obtained using modal damping and FRF obtained using damping parameters (Updated)

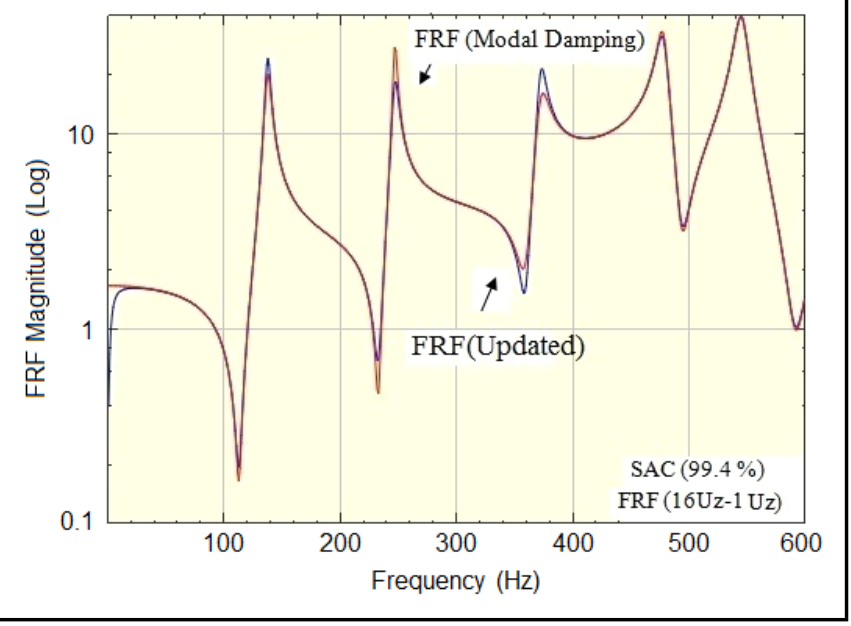

Figure 3. Comparison of trial and regenerated FRFs using updated parameters for a typical cross FRF.

to get converged values of the modal properties. The numerical FRFs were synthesized from the undamped modal properties, using the modal damping factors from experimental observation. The mode shapes and initial MAC values for correlations between modes are not shown for brevity. Differences in modal and FRFs between the experimental observation and the finite element model were resolved through model updating after selecting appropriate sets of parameters. The most likely parameters affecting the results are 1) the in-plane material constants affecting the resonant frequency shifts and 2) the damping factors affecting the response amplitude sharpness of the FRF curves of the pultruded FRP plate.

First, the material parameters were updated using the IEM considering the experimental modal data, viz. frequencies and mode shapes. The convergence curves from a set of selected initial values of the parameters are shown in Fig. 6.

Frequencies obtained using updated elastic material parameters along with the experimental frequencies are shown in Table 3. The experimental modal damping factors are also shown.

The final MAC values indicate good correlations between the updated mode shapes and the observed mode shapes. Since MAC is a global vector correlation function at resonant fre- 


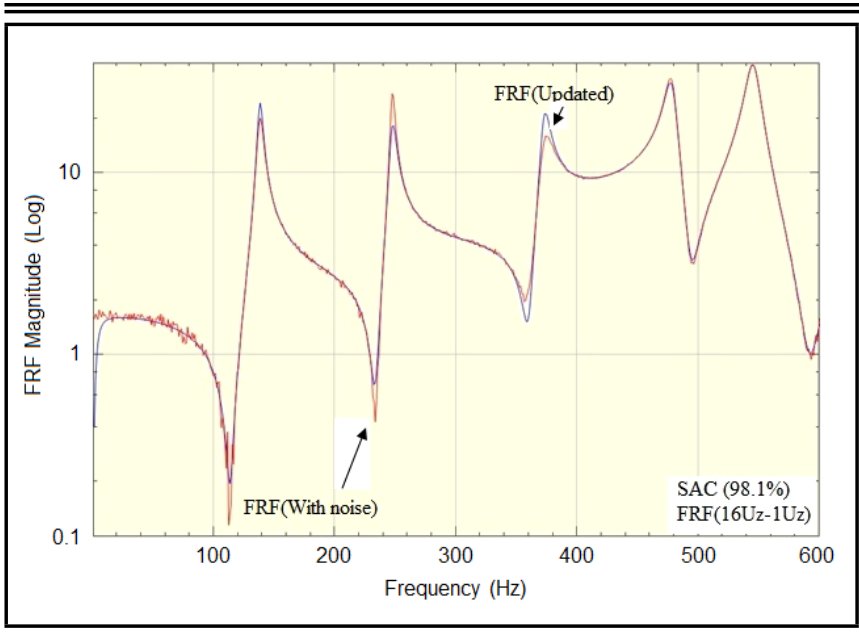

Figure 4. Comparison of actual FRF (with $2 \%$ noise) and regenerated FRF using updated parameter values.

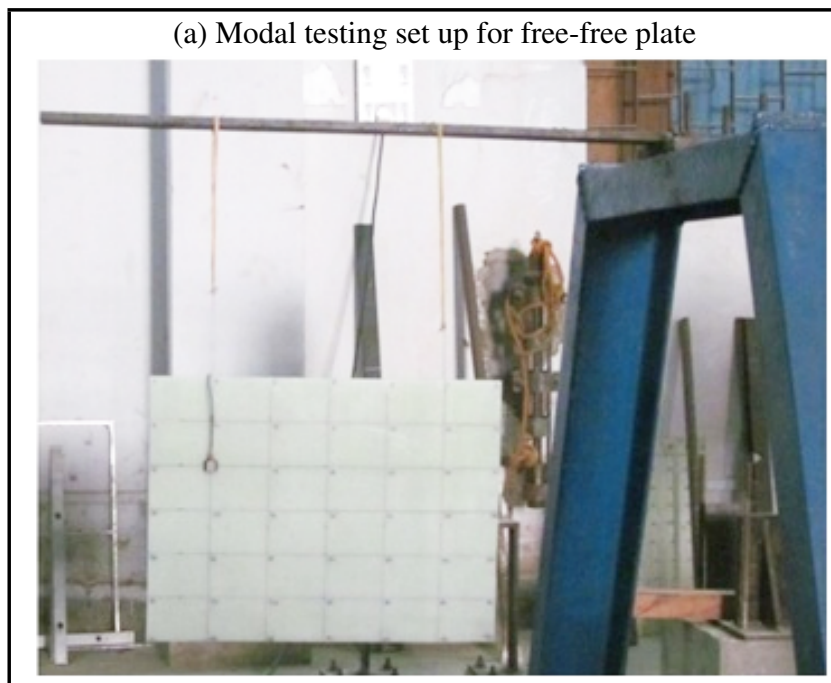

(b) Grid point for measurement of FRFs

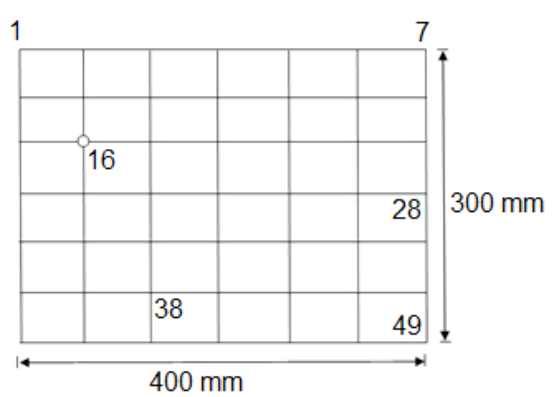

Figure 5. (a) Experimental setup for modal test, (b) Grid points for measurement of FRFs of the plate.

quencies, it does not immediately indicate the level of variations of FRF magnitudes at intermediate frequencies. To gain insight into the benefit of the global updating process, CSAC and CSF were plotted (Fig. 7) with respect to the frequencies, which indicate excellent correlations except near a few antiresonant points.

The average material constants determined experimentally, were $E_{x}=33.05 \mathrm{GPa}, E_{y}=31.80 \mathrm{GPa}, G_{x y}=5.73 \mathrm{GPa}$, and $v_{x y}=0.15$; the updated material constants were found to be $E_{x}=35.64 \mathrm{GPa}, E_{y}=32.31$, and $G_{x y}=7.12 \mathrm{GPa}$ respectively. The updated material parameters differed only slightly from the results obtained by updating a beam speci- (a) Convergence of $E_{y}$

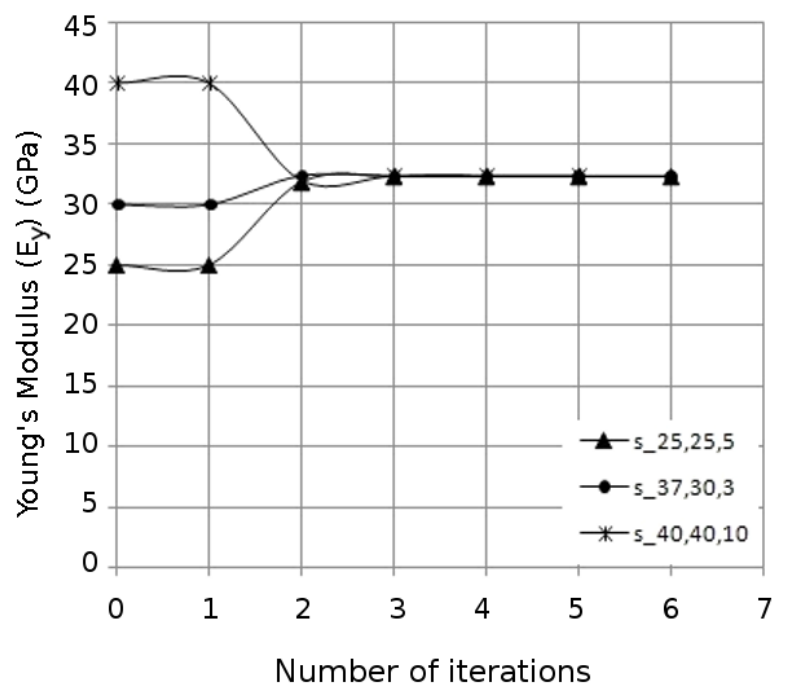

(b) Convergence of $G_{x y}$

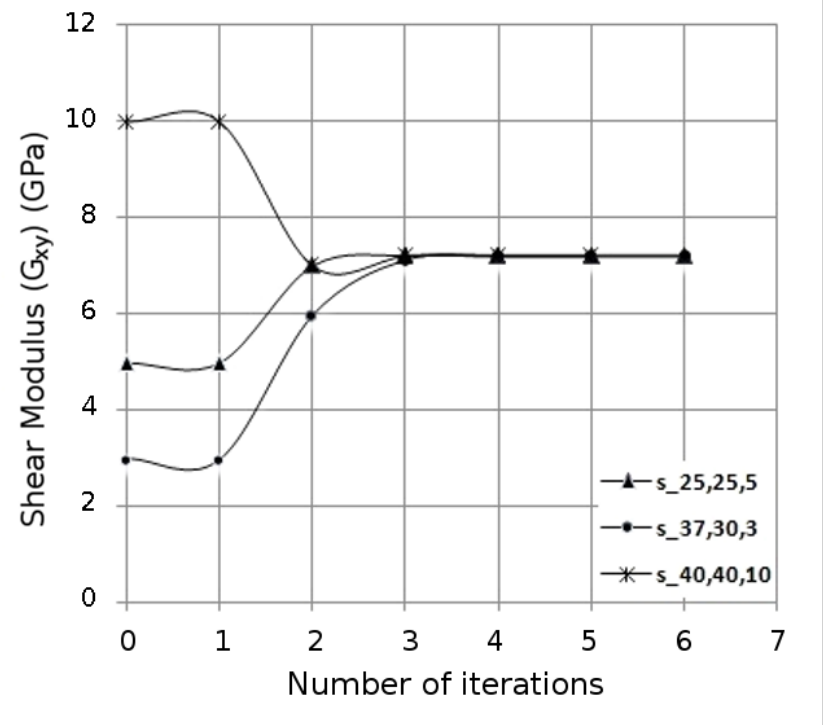

Figure 6. Typical convergence curves for material parameters from selected initial values.

Table 3. Updated and measured eigenvalues and damping.

\begin{tabular}{|c|c|c|}
\hline Updated freq. (Hz) & Exp. freq. (Hz) & Measured damping (\%) \\
\hline 164.11 & 164.15 & 3.247 \\
256.53 & 256.59 & 1.8351 \\
418.57 & 420.1 & 1.7126 \\
481.92 & 482.27 & 1.1702 \\
576.47 & 577.30 & 1.2725 \\
710.41 & 710.9 & 1.0058 \\
\hline
\end{tabular}

men, and can be attributed to the size effects of the beam sample. The updated parameters obtained from the updating exercise of the plate specimen are believed to be more accurate. Table 3 shows the regenerated frequencies, experimentally observed frequencies, and the experimental modal damping factors.

Next, the damping parameters were updated. The initial values of these parameters were selected again from Eq. (7), following the methodology outlined in the numerically simulated example. The two sets of estimated damping coefficients shown in Table 4, along with two arbitrary values, were used 
(a) MAC for FE modes and experimentally obtained modes

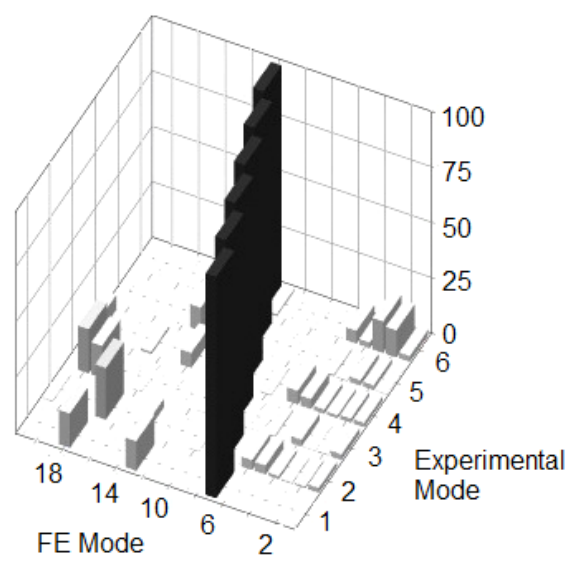

(b)

Variation of CSAC and CSF

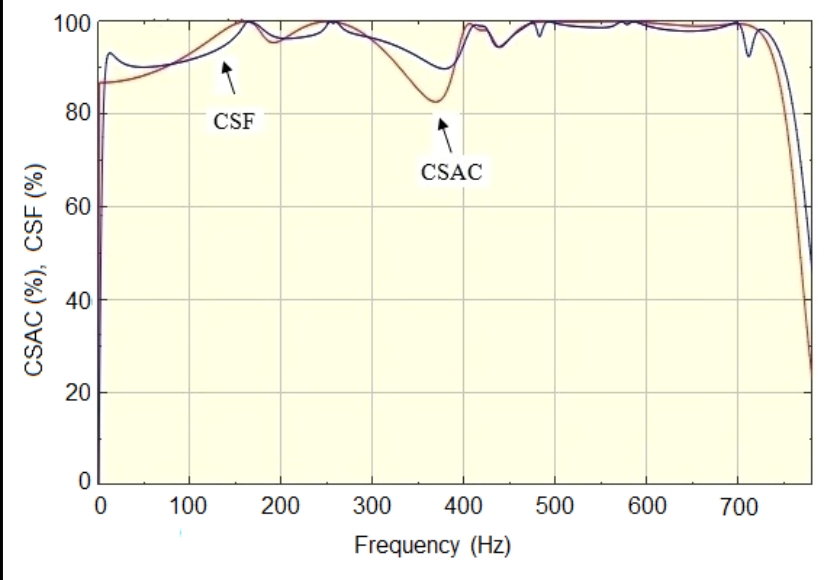

Figure 7. (a) MAC value of different mode, (b) CSF and CSAC along frequency.

Table 4. Initial value of damping coefficients from experimentally obtained modal damping.

\begin{tabular}{|c|c|c|c|c||}
\hline & $\begin{array}{c}\text { Mode considered } \\
\text { for average } \\
\text { of } \omega_{1} \text { and } \zeta_{1}\end{array}$ & $\begin{array}{c}\text { Mode considered } \\
\text { for average } \\
\text { of } \omega_{1} \text { and } \zeta_{1}\end{array}$ & $a_{0}$ & $a_{1}$ \\
\hline Trial_1 & 1,2 & 3,4 & 10.69 & $2.95 \mathrm{E}-5$ \\
Trial_2 & $1,2,5$ & $3,4,5$ & 12.54 & $1.40 \mathrm{E}-5$ \\
Trial_3 & - & - & 100 & $3.00 \mathrm{E}-6$ \\
Trial_4 & - & - & 90.00 & $4.00 \mathrm{E}-6$ \\
\hline
\end{tabular}

to test the robustness of the algorithm.

FRFs using a typical set of trial values and updated values of damping parameters are shown in Fig. 8. The regenerated responses using the updated damping parameters matched well with the experimentally obtained FRFs and added confidence to the updating procedures. In Fig. 9, the comparison between the regenerated FRFs using modal damping values and the updated damping parameters were found to be almost exactly matching. The SAC value also approaches 1 , indicating favourable global correlations.

The corresponding values of the real and imaginary parts of the FRFs computed using updated Rayleigh damping coefficients are shown in Fig. 10(a) and 10(b) respectively.

The convergence curves of the damping parameters are shown in Fig. 11, and were found to be monotonically converging in all cases, with the final updated parameters being $a_{0}=72.3$ and $a_{1}=0.38 \mathrm{E}-5$ respectively. Altogether, 6 modes were considered. It was immediately apparent that the (a) Comparison of FRF obtained experimentally and FRF obtained using damping parameters (Trial_1)

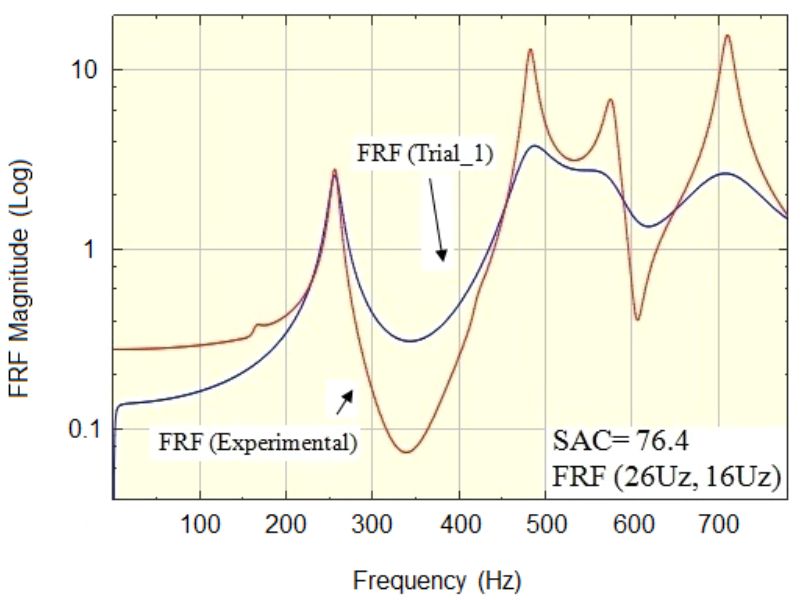

(b) Comparison of FRFs obtained experimentally and FRF obtained using damping parameters (Updated)

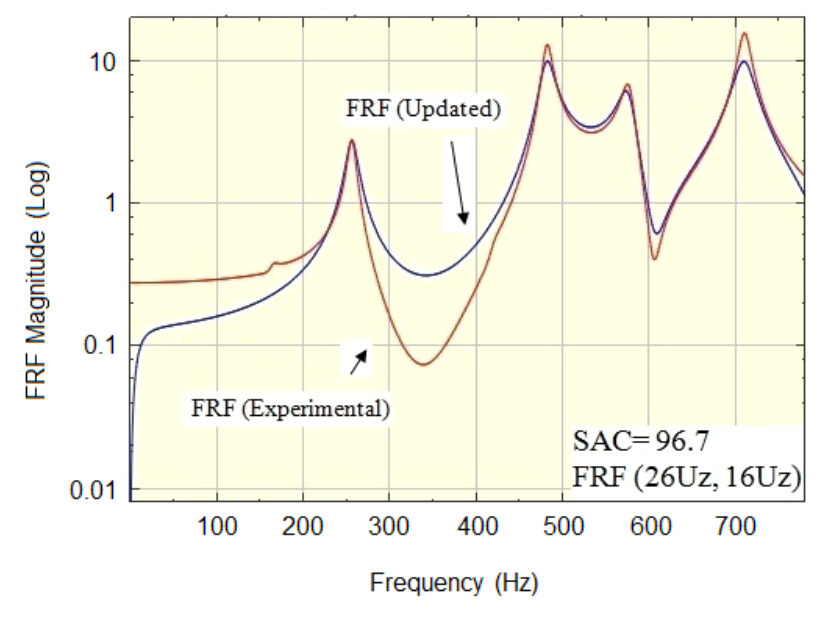

Figure 8. Comparison between experimental FRF and FRF using Rayleigh damping parameters.

Table 5. Updated damping coefficients for different frequency ranges.

\begin{tabular}{|c|c|c|}
\hline Frequency range up to & Updated $a_{0}$ & Updated $a_{1}$ \\
\hline $450 \mathrm{~Hz}$ & $7.97 \mathrm{E} 1$ & $2.29 \mathrm{E}-6$ \\
$650 \mathrm{~Hz}$ & $7.45 \mathrm{E} 1$ & $3.93 \mathrm{E}-6$ \\
\hline
\end{tabular}

parameters converged to values much different from the initial estimate in Eq. (7); this can be attributed to the effect of incorporating more than two frequencies at a time.

As a verification exercise, experimentally observed FRFs that had not been used for updating were compared with corresponding regenerated FRFs using the updated parameters. The correlations were also found to be favourable, though these results are not shown for brevity. The results of this exercise help confirm the robustness of the current algorithm.

The question not yet resolved is the acceptable number of modes to include in the determination of a globally representative set of damping parameters. Convergence of the damping parameters with increasing numbers of modes - up to 8 modes in the current model updating exercise-indicated fewer variations amongst themselves as shown in Table 5. There were difficulties in accurately measuring higher modes during this investigation, thus results are hence not shown here.

A higher value of the mass-proportional coefficients in 
(a) Comparison of FRFs obtained experimentally and FRFs obtained using modal damping

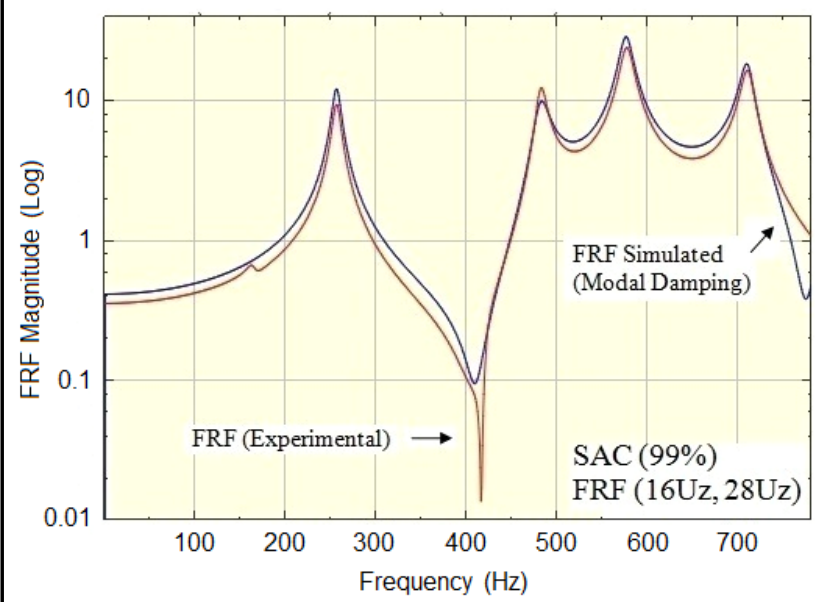

(b) Comparison of FRFs obtained experimentally and FRFs obtained using damping parameters (Updated)

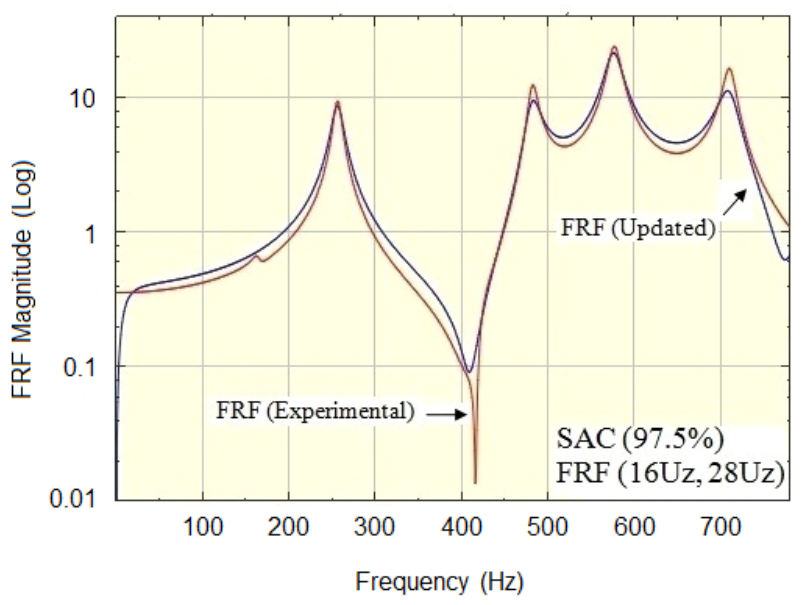

Figure 9. Comparison between experimental FRF and FRFs obtained using modal damping factor and Rayleigh damping parameters.

Rayleigh damping indicates that the equivalent viscous damping effect is predominating and can be modelled conveniently with assumptions about velocity proportional damping. On the other hand, higher values in stiffness proportional coefficients indicate that damping is mainly due to internal material damping, and may be nonviscous in nature. In this experimental investigation, the updated mass proportional damping coefficient $a_{0}$ was indeed found to be of a much higher order than the stiffness proportional damping coefficient. Subsequently, the updated damping parameters $a_{0}$ and $a_{1}$ can be used to compute the modal damping coefficients. In the present investigation, the modal damping factor was overestimated in comparison to the measured one (Fig. 12). Estimations of relaxation factors corresponded to the measured modal damping. The value of $\mu_{0}$ was found to be $95 \mathrm{~s}^{-1}$ and the value of $\mu_{1}$ was estimated as $150000 \mathrm{~s}^{-1}$, which closely resembles viscous damping in the present case study. Additionally, estimated lower values of $\mu_{0}$ reaffirm the viscous damping model. ${ }^{16}$ For a structure in which internal material damping force is dominant, the damping coefficients of the present model can conveniently be adjusted through model updating to produce accurate vibration responses. (a) Variation of real part of FRF

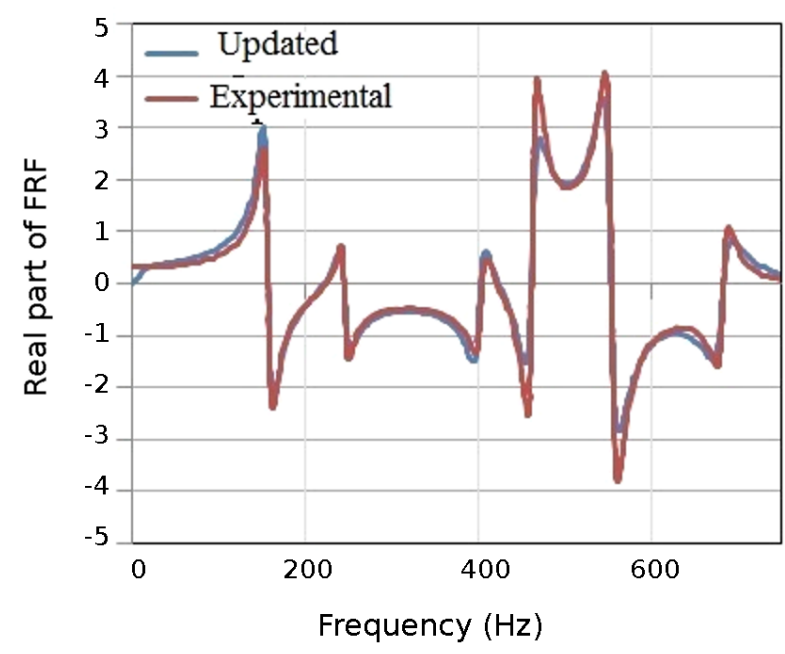

(b) Variation of imaginary part of FRF)

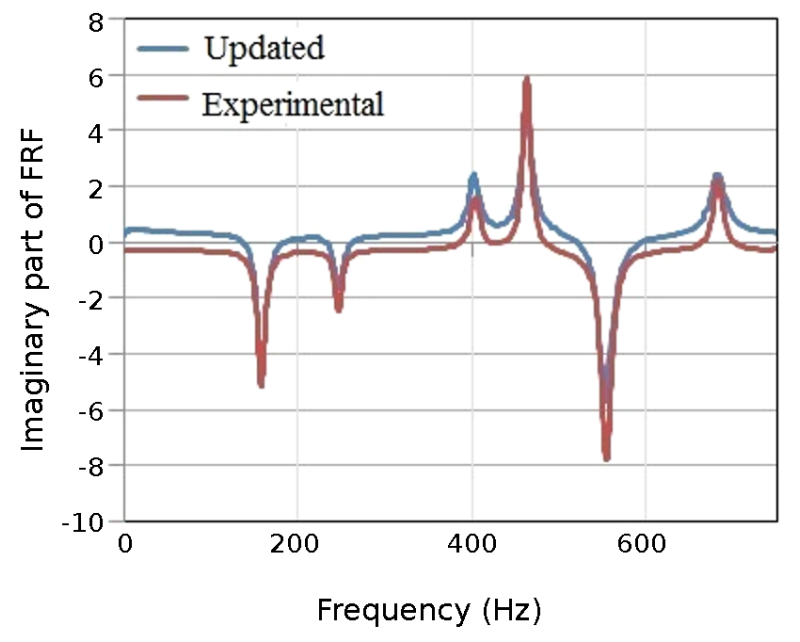

Figure 10. (a) Real and (b) Imaginary part of the FRF computed using updated Rayleigh damping coefficients.

\section{CONCLUSIONS}

A finite element model updating algorithm was implemented to estimate proportional damping parameters of a fibre reinforced plastic plate over a large frequency range from experimentally-observed modal and frequency response functions. In most practical cases of existing fibre reinforced plastic structures, it will be mandatory to update the uncertain material parameters first, followed by estimation of damping parameters. The number of frequencies to include will be decided depending upon the frequencies of interest, but includes all frequencies contributing effectively to the response functions. For updating material parameters, information from frequencies and mode shapes is convenient; alternatively, for updating damping parameters, frequency response functions are more appropriate.

In this study a numerically-simulated example was presented, followed by a detailed experimental investigation of an FRP composite plate under free-boundary conditions for the correct estimation of the elastic parameters and damping coefficients together. The results were verified using measured responses which are not used during updating, thereby eliminat- 


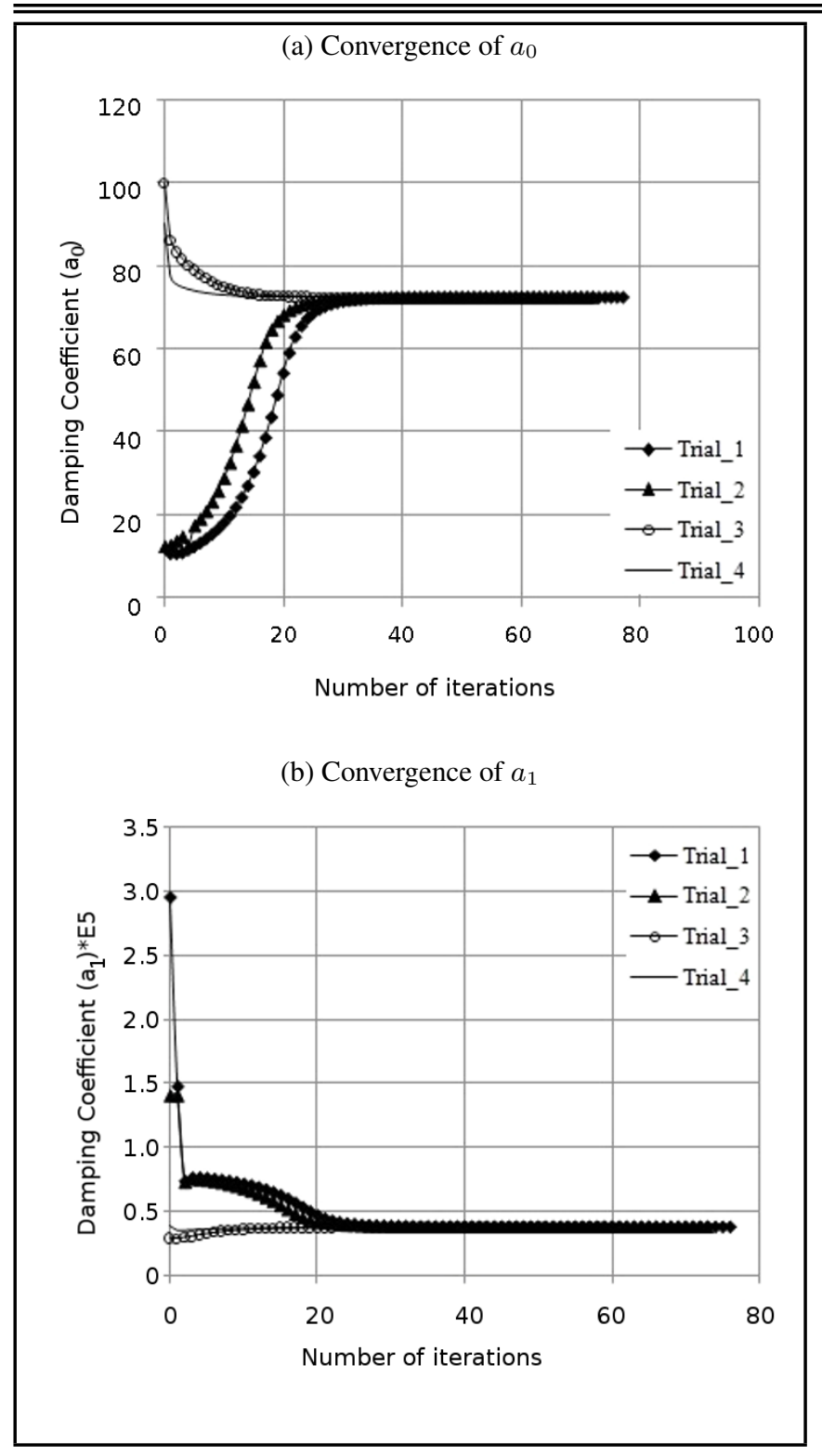

Figure 11. Convergence curve for damping parameter.

ing the possibility of the updating methodology being biased towards limited measured observations. Finally, the updated material parameters were checked through static characterization tests.

The relationship between the viscous damping and nonviscous damping was also established through an estimation of a pair of relaxation parameters using model updating. The actual damping effect for this experimental case study was found to be predominantly viscous. The methodology used in this study can easily be applied to model the damping effects of plates where internal material damping is dominating, instead of the viscous effects by adjustment of the damping relaxation parameters through model updating.

\section{REFERENCES}

1 Gaul, L. Tutorial guideline VDI 3830: Damping of materials and members, IMAC XXVII: Conference and Exposition on Structural Dynamics, (2009). https://dx.doi.org/10.1007/978-1-4419-9834-7_14

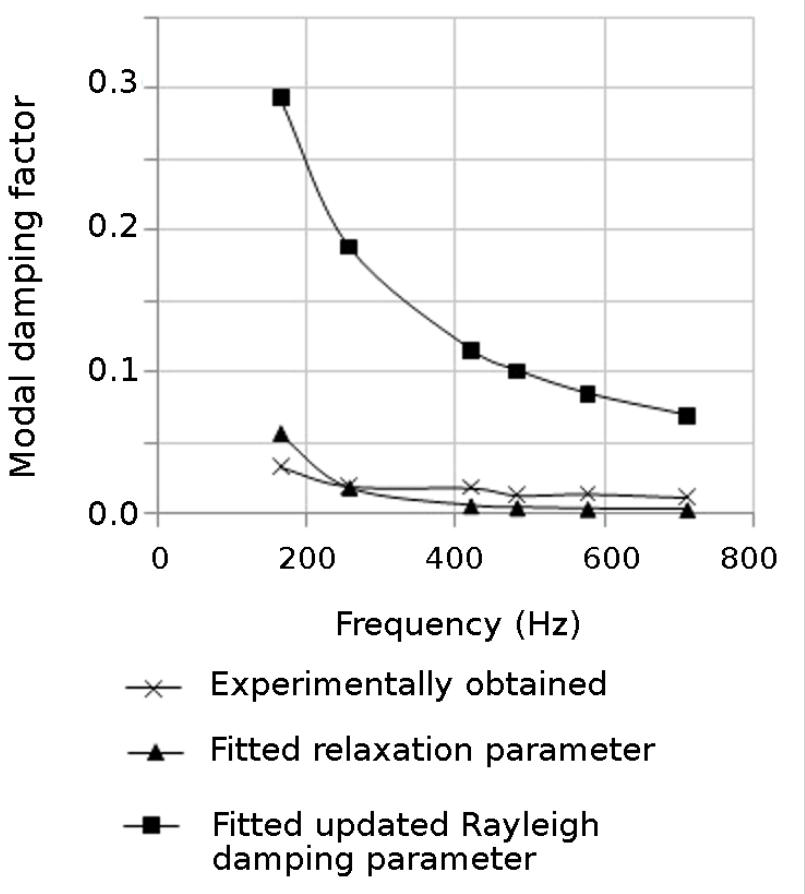

Figure 12. Variation of modal damping factor with frequency.

2 Rayleigh, L. Theory of sound (two volumes), Dover Publications, (1877).

3 Caughey, T. K. Classical normal modes in damped linear dynamic systems, Journal of Applied Mechanics, 27 (2), 269-271, (1960). https://dx.doi.org/10.1115/1.3643949

4 Caughy, T. K. and O'Kelly, M. E. J. Classical normal modes in damped linear dynamic systems, Journal of Applied Mechanics, 32 (3), 583-588, (1965). https://dx.doi.org/10.1115/1.3627262

5 Clough, R. W. and Penzien, J. Dynamics of structures, 2nd Rev. Ed., McGraw Hill, New York, (1993).

6 Woodhouse, J. Linear damping models for structural vibration, Journal of Sound and Vibration, 215 (3), 547-569, (1998). https://dx.doi.org/10.1006/jsvi.1998.1709

7 Adhikari, S. Damping modelling using generalized proportional damping, Journal of Sound and Vibration, 293 (1-2), 156-170, (2006). https://dx.doi.org/10.1016/j.jsv.2005.09.034

8 Adhikari, S. and Phani, A. S. Experimental identification of generalized proportional viscous damping matrix, Journal of Vibration and Acoustics, 131 (1), (2009). https://dx.doi.org/10.1115/1.2980400

9 Lee, J. H. and Kim, J. Development and validation of a new experimental method to identify damping matrices of a dynamic system, Journal of Sound and Vibration, 246 (3), 505-524, (2001). https://dx.doi.org/10.1006/jsvi.2001.3670

10 Arora, V. Structural damping identification method using normal FRFs, International Journal of 
Solid and Structures, 51 (1), 133-143, (2014). https://dx.doi.org/10.1016/j.ijsolstr.2013.09.017

11 Chen, S. Y. J., Ju, M. S., and Tsuei, Y. G. Estimation of mass, stiffness and damping matrices form frequency response functions, Journal of Vibration and Acoustics, 118, 78-82, (1996). https://dx.doi.org/10.1115/1.2889638

12 Fritzen, C. P. Identification of mass, damping, and stiffness matrices of mechanical systems, Journal of Vibration, Acoustics Stress and Reliability in Design, 108 (1), 9-16, (1986). https://dx.doi.org/10.1115/1.3269310

13 Reix, C. C. T., Gerard, A., and Dascotte, E. Updating the damping matrix using frequency response data, 14th International Modal Analysis Conference, (1996).

14 Arora, V., Singh, S. P., and Kundra, T. K. Finite element model updating with damping identification, Journal of Sound and Vibration, 324 (3-5), 1111-1123, (2009). https://dx.doi.org/10.1016/j.jsv.2009.02.048

15 Friswell, M. I., Inman, D. J., and Pilkey, D. F. The direct updating of damping and stiffness matrices, AIAA Journal, 36, 491-493, (1998). https://dx.doi.org/10.2514/3.13851

16 Pan, Y. and Wang, Y. Iterative method for exponential damping identification, Computer-Aided Civil and Infrastructure Engineering, 30 (3), 229-243, (2015). https://dx.doi.org/10.1111/mice.12077

17 Kausel, E. Damping matrices revisited, Journal of Engineering Mechanics, 140 (8), (2014). https://dx.doi.org/10.1061/(asce)em.1943-7889.0000770

18 Dalenbring, M. Damping function estimation based on measured vibration frequency responses and finiteelement displacement modes, Mechanical Systems and Signal Processing, 13 (4), 547-569, (1999). https://dx.doi.org/10.1006/mssp.1999.1221

19 Dalenbring, M. An explicit formulation of a threedimensional material damping model with transverse isotropy, International Journal of Solids and Structures, 39 (1), 225-249, (2002). https://dx.doi.org/10.1016/s00207683(01)00158-5

20 Dalenbring, M. Experimental material damping estimation for planar isotropic laminate structures, International Journal of Solids and Structures, 39 (19), 5053-5079, (2002). https://dx.doi.org/10.1016/s0020-7683(02)00343-8

21 Dalenbring, M. Validation of estimated isotropic viscoelastic material properties and vibration response prediction, Journal of Sound and Vibration, 265 (2), 269-287, (2003). https://dx.doi.org/10.1016/s0022-460x(02)01261-0

22 Dovstam, K. Augmented Hooke's law in frequency domain. A three dimensional, material damping formulation, International Journal of Solids and Structures, 32 (19), 2835-2852, (1995). https://dx.doi.org/10.1016/0020$7683(94) 00269-3$
23 Dovstam, K., Goransson, P., and Gartmeier, O. On linear modeling of interface damping in vibrating structures, Journal of Sound and Vibration, 331 (19), 4299-4312, (2012). https://dx.doi.org/10.1016/j.jsv.2012.03.036

$24 \mathrm{Li}$, Z. and Crocker, M. J. A review on vibration damping in sandwich composite structures, International Journal of Acoustics and Vibration, 10 (4), 159-169, (2005). https://dx.doi.org/10.20855/ijav.2005.10.4184

25 Gelman, L., Jenkin, P., and Petrunin, I. Vibroacoustical damping diagnostics: Complex frequency response function versus its magnitude, International Journal of Acoustics and Vibration, 11 (3), 120-124, (2006). https://dx.doi.org/10.20855/ijav.2006.11.3195

26 Akrout, A., Hammami, L., Karra, C., Tahar, M. B., and Haddar, M. Vibroacoustic damping simulation of two laminated glass panels coupled to viscothermal fluid layer, International Journal of Acoustics and Vibration, 15 (2), 7990, (2010). https://dx.doi.org/10.20855/ijav.2010.15.2261

$27 \mathrm{Wu}$, J. W. and Huang, L. Z. Natural frequencies and acoustic radiation mode amplitudes of laminated composite plates based on the layerwise FEM, International Journal of Acoustics and Vibration, 18 (3), (2013). https://dx.doi.org/10.20855/ijav.2013.18.3328

28 Imregun, M., Sanliturk, K. Y., and Ewins D. J. Finite element model updating using frequency response function data: II. Case study on a medium-size finite element model, Mechanical Systems and Signal Processing, 9 (2), 203-213, (1995). https://dx.doi.org/10.1006/mssp.1995.0016

29 Collins, J. D., Hart, G. C., Hasselman, T. K., and Kennedy B. Statistical identification of structure, AIAA Journal, 12 (2), 185-190, (1974). https://dx.doi.org/10.2514/3.49190

30 Mondal, S. and Chakraborty, S. An inverse approach for the determination of viscous damping model of fibre reinforced plastic beams using finite element model updating, International Journal of Acoustics and Vibration, 21 (1) ,93-102, (2016). https://dx.doi.org/10.20855/ijav.2016.21.1399

31 Ewins ,D. J. Modal testing: Theory, practice and application, London, England, (2000).

32 Bathe, K. J. Finite element procedures, New Delhi: PHI, (2010).

33 Allemang, R. J. The modal assurance criterion-Twenty years of use and abuse, Sound \& Vibration, 37, 14-23, (2003).

34 Dascottee, E. and Strobbe, J. Updating finite element models using FRF correlation functions, IMAC XVII-17th International Modal Analysis Conference, (1999).

35 Adhikari, S. Damping models for structural vibration, $\mathrm{PhD}$ Thesis, Cambridge University, (2000). 